\title{
Detection of a Coherent Longitudinal Optical Phonon in a GaAs Buffer Layer Optically Covered with a GaSb Top Epitaxial Layer Using Terahertz Spectroscopy
}

Hideo Takeuchi, Syuichi Tsuruta, Masaaki Nakayama

\begin{tabular}{|c|l|}
\hline Citation & AIP Conference Proceedings, 1416(1): 84-87 \\
\hline Issue Date & $2011-12-28$ \\
\hline Type & Conference paper \\
\hline Textversion & publisher \\
\hline Right & $\begin{array}{l}\text { C American Institute of Physics. This article may be downloaded for personal use only. } \\
\text { Any other use requires prior permission of the author and AIP Publishing. The following } \\
\text { article appeared in AIP Conference Proceedings and maybe found at } \\
\text { https://doi.org/10.1063/1.3671704 }\end{array}$ \\
\hline DOI & \begin{tabular}{l}
$10.1063 / 1.3671704$ \\
\hline
\end{tabular} \\
\hline
\end{tabular}

Self-Archiving by Author(s)

Placed on: Osaka City University Repository

TAKEUCHI H., TSURUTA S., \& NAKAYAMA M. (2011). Detection of a coherent longitudinal optical phonon in a GaAs buffer layer optically covered with a GaSb top epitaxial layer using terahertz spectroscopy. AIP Conference Proceedings. 1416, 84-87. 


\section{AIP $\mid$ Conference Proceedings}

Detection of a Coherent Longitudinal Optical Phonon in a GaAs Buffer Layer Optically Covered with a GaSb Top Epitaxial Layer Using Terahertz Spectroscopy

Hideo Takeuchi, Syuichi Tsuruta, and Masaaki Nakayama

Citation: AIP Conf. Proc. 1416, 84 (2011); doi: 10.1063/1.3671704

View online: http://dx.doi.org/10.1063/1.3671704

View Table of Contents: http://proceedings.aip.org/dbt/dbt.jsp?KEY=APCPCS\&Volume=1416\&Issue $=1$

Published by the American Institute of Physics.

\section{Related Articles}

Deep levels in $\mathrm{H}$-irradiated GaAs1-xNx $(\mathrm{x}<0.01)$ grown by molecular beam epitaxy J. Appl. Phys. 110, 124508 (2011)

X-ray magnetic circular dichroism of ferromagnetic Co4N epitaxial films on $\mathrm{SrTiO3(001)} \mathrm{substrates} \mathrm{grown} \mathrm{by}$ molecular beam epitaxy

Appl. Phys. Lett. 99, 252501 (2011)

Carrier dynamics and activation energy of CdTe quantum dots in a CdxZn1xTe quantum well

Appl. Phys. Lett. 99, 231908 (2011)

Demonstration of forward inter-band tunneling in GaN by polarization engineering

Appl. Phys. Lett. 99, 233504 (2011)

X-ray reciprocal space mapping of dislocation-mediated strain relaxation during InGaAs/GaAs(001) epitaxial growth

J. Appl. Phys. 110, 113502 (2011)

\section{Additional information on AIP Conf. Proc.}

Journal Homepage: http://proceedings.aip.org/

Journal Information: http://proceedings.aip.org/about/about_the_proceedings

Top downloads: http://proceedings.aip.org/dbt/most_downloaded.jsp?KEY=APCPCS

Information for Authors: http://proceedings.aip.org/authors/information_for_authors

\section{ADVERTISEMENT}

Explore AIP's new open-access journal

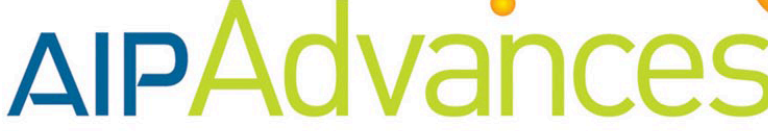

Article-level metrics now available

Join the conversation! Rate \& comment on articles 


\title{
Detection of a Coherent Longitudinal Optical Phonon in a GaAs Buffer Layer Optically Covered with a GaSb Top Epitaxial Layer Using Terahertz Spectroscopy
}

\author{
Hideo Takeuchi ${ }^{\mathrm{a}}$, Syuichi Tsuruta ${ }^{\mathrm{b}}$, and Masaaki Nakayama ${ }^{\mathrm{b}}$ \\ ${ }^{a}$ Department of Electronic Systems Engineering, School of Engineering, The University of Shiga Pre- \\ fecture, 2500 Hassaka-cho, Hikone, Shiga 522-8533, Japan \\ ${ }^{b}$ Department of Applied Physics, Graduate School of Engineering, Osaka City University, 3-3-138 \\ Sugimoto, Sumiyoshi-ku, Osaka 558-8585, Japan
}

\begin{abstract}
We demonstrate that, in a $\mathrm{GaSb} / \mathrm{GaAs}$ epitaxial structure, the coherent longitudinal optical (LO) phonon in the GaAs layer optically covered with the GaSb top layer is observed utilizing terahertz spectroscopy. In the terahertz-wave measurement, the Fourier power spectrum of the terahertz waveform exhibits both the GaAs and the GaSb LO phonons; namely, the coherent LO phonon in the optically covered GaAs buffer layer is observed in the terahertz-wave measurement. This fact demonstrates that the instantaneous surface potential modulation originating from the impulsive carrier excitation by the pump pulses reaches the GaAs buffer layer. This surface potential modulation generates the coherent GaAs LO phonon.
\end{abstract}

Keywords: Terahertz electromagnetic wave; Coherent longitudinal optical phonon; GaSb; GaAs. PACS: 78.47.-p; 78.47.J-; 78.66.Fd

\section{INTRODUCTION}

Terahertz $(\mathrm{THz})$ spectroscopy has been making a remarkable progress owing to the development of femtosecond pulse laser systems. ${ }^{1,2}$ The above-mentioned progress enables the detection of the $\mathrm{THz}$ electromagnetic waves from coherent phonons. ${ }^{3-7}$ $\mathrm{THz}$ spectroscopy gives an interesting issue on the generation of the coherent phonons. In the conventional Raman scattering process, phonons are generated directly by the excitation light. In the emission process of the $\mathrm{THz}$ wave from longitudinal optical (LO) phonons, the coherent LO phonon is initially induced by the surge current of the photogenerated carriers that leads to the change in the band bending ${ }^{8}$ through the screening effect. ${ }^{9}$ The above-mentioned THz-wave emission mechanism suggests that the substantial trigger of driving the coherent phonon is not the pump-beam illumination but the resultant photogenerated carriers. Accordingly, the appearance of the following phenomenon is expected: Even in the case where a given layer is optically covered with an upper layer, the $\mathrm{THz}$ wave from the coherent LO phonons is generated through a change in the band bending.

In the present work, we explore the feasibility of generating the $\mathrm{THz}$ wave from the coherent GaAs LO phonon in the buffer layer optically covered with a GaSb top layer.

15th International Conference on Narrow Gap Systems (NGS15)

AIP Conf. Proc. 1416, 84-87 (2011); doi: 10.1063/1.3671704

(C) 2011 American Institute of Physics 978-0-7354-0993-4/\$30.00 


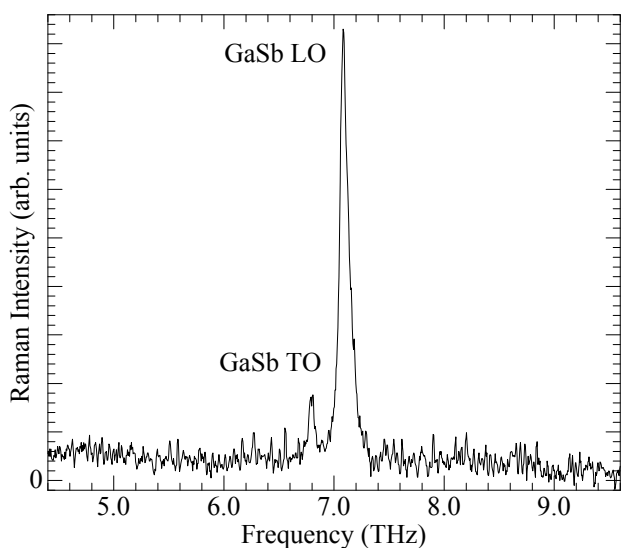

FIGURE 1. Raman scattering spectrum of the $\mathrm{GaSb} / \mathrm{GaAs}$ structure measured at RT.

It is demonstrated that the $\mathrm{THz}$ wave from the coherent GaAs LO phonon in the buffer layer are generated. We discuss the generation mechanism of the coherent phonon in terms of the instantaneous modulation of the surface potential bending.

\section{EXPERIMENTAL PROCEDURES, RESULTS, AND DISCUSSION}

The present sample, the undoped $\mathrm{GaSb}$ /undoped GaAs epitaxial structure, was grown on a (001)-oriented semi-insulating GaAs substrate by molecular beam epitaxy. The thicknesses of the GaSb top layer and GaAs buffer layer were $900 \mathrm{~nm}$ and 200 $\mathrm{nm}$, respectively. Initially, we performed the Raman scattering measurement at room tem-perature (RT). The photon energy and power of the excitation beam were $1.58 \mathrm{eV}$ and $19 \mathrm{~mW}$, respectively. Figure 1 shows the Raman scattering spectrum of the present sample. Two bands are observed at the frequencies of 6.8 and $7.1 \mathrm{THz}$. Taking ac-count of the fact that the frequencies of the GaSb transverse optical (TO) phonon and LO phonon are 6.8 and $7.0 \mathrm{THz}$, respectively, the observed Raman bands are assigned to the GaSb TO and LO phonons in order of frequency. The GaAs LO phonon with a frequency of $8.8 \mathrm{THz}$ is not observed in the Raman spectrum. This is because the pen-etration length of the excitation beam is $160 \mathrm{~nm}$ in $\mathrm{GaSb} ;{ }^{10}$ namely, the GaSb top layer optically covers the GaAs buffer layer.

The $\mathrm{THz}$ waves were measured at RT using laser pulses with a duration time of about 50 fs. The pump beam was focused on the sample with the angle of incidence of $45^{\circ}$. The emitted $\mathrm{THz}$ wave was detected by a photoconductive dipole antenna with a gap of $6 \mu \mathrm{m}$ formed on a low-temperature grown GaAs layer. The powers of the pump and gate beams were 120 and $10 \mathrm{~mW}$, respectively. The photon energies of both the beams were the same: $1.57 \mathrm{eV}$. The scan range of the time delay was -2 to $8 \mathrm{ps}$, so that the time window was $10 \mathrm{ps}$. Taking account of the fact that the period of the GaAs LO phonon is $113 \mathrm{fs}$, the time window of $10 \mathrm{ps}$ is enough to resolve the coherent LO phonon band in the Fourier power spectrum of the THz waveform.

The THz waveform is shown in Fig. 2(a). Around the time delay of 0 ps, the monocycle oscillation pulse, the so-called first burst resulting from the surge current, appears. The first burst is followed by the oscillatory profile with a beat pattern. The appearance of the beat indicates that multiple oscillation modes are observed. In order to 

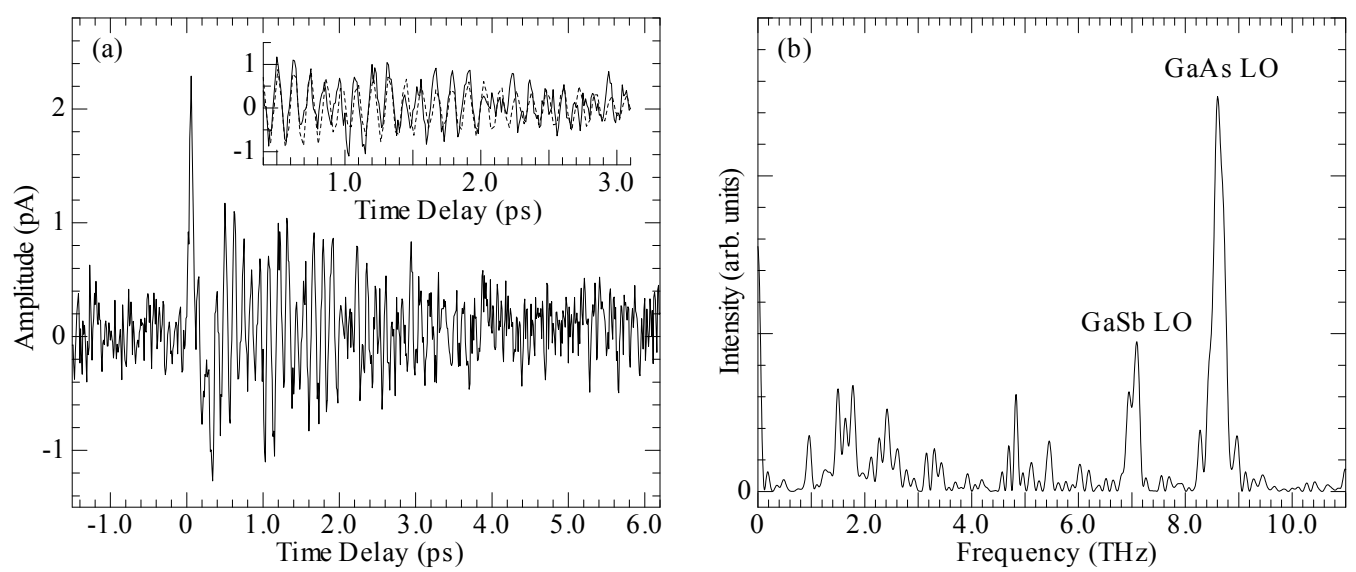

FIGURE 2. (a) Amplitude of the $\mathrm{THz}$ waveform of the $\mathrm{GaSb} / \mathrm{GaAs}$ structure as a function of time delay at RT. The inset shows the oscillatory profile between 0.5 and $3.0 \mathrm{ps}$ in order to highlight the beat pattern. The dashed curve in the inset is the fitting results with use of two damped sinusoidal functions. (b) Fourier power spectrum of the THz waveform shown in Fig. 2(a).

clarify the observed mode, we performed the Fourier transform. The Fourier power spectrum is shown in Fig. 2(b). The band of the first burst weakly appears around 2.0 $\mathrm{THz}$. The band at 7.0 THz is assigned to the coherent GaSb LO phonon. It is emphasized that the GaAs LO phonon band emerges at $8.6 \mathrm{THz}$ in the Fourier power spectra of the THz wave. Taking account of the fact that the frequency of the GaAs LO phonon in a single crystal is $8.8 \mathrm{THz}$, the shift of the phonon frequency $\omega \Delta$ is $-0.2 \mathrm{THz}$ in the present GaAs buffer layer. The value of $\omega \Delta$ corresponds to the tensile strain of 1.8 $\times 10^{-2}$, assuming the biaxial strain induced by the lattice mismatch. In the estimation of the strain, the phonon deformation potential was taken from Ref. 11. Thus, we conclude that the THz wave from the coherent GaAs LO phonons is observable even in the case where the GaAs buffer layer is optically covered with the GaSb layer. Here, it is noteworthy that the intensity of the coherent LO phonon band is relatively large, in spite of the fact that the sensitivity of the photoconductive dipole antenna remarkably drops in the frequency range higher than $\sim 4 \mathrm{THz}$. This fact phenomenologically indicates that coherent LO phonons have a potential of being strong $\mathrm{THz}$ emitters. In fact, the intense $\mathrm{THz}$ emission from the coherent GaAs LO phonon is also observed with the photoconductive dipole antenna in GaAs/AlAs multiple quantum wells ${ }^{6}$ and undoped GaAs/n-type GaAs epitaxial structures. ${ }^{7}$

We also performed the fitting to the $\mathrm{THz}$ waveform with use of two damped sinusoidal functions in order to confirm the appearance of the two LO-phonon bands in the Fourier power spectrum shown in Fig. 2(b). The solid curve in the inset of Fig. 2(a) depicts the observed oscillatory profile in the time region from 0.4 to $3.1 \mathrm{ps}$. Since the oscillatory profile in the longer time region is noisy, we exclude it in the fitting. The dashed curve in the inset of Fig. 2(a) indicates the result of the above-mentioned fitting, where the fitting parameters used are as follows: The LO-phonon frequencies of $\mathrm{GaSb}$ and GaAs are 7.1 and 8.6 THz, respectively, and the decay times of the $\mathrm{GaSb}$ and GaAs LO phonons are 4.0 and $3.1 \mathrm{ps}$, respectively. The fitting curve almost agrees with the experimental $\mathrm{THz}$ waveform in spite of the fluctuation of the background signal. Accordingly, the present Fourier transform analysis is confirmed to be appropriate. 
Finally, we briefly discuss the responsible factor of the above-mentioned phenomenon. As pointed out in INTRODUCTION, the pump-beam illumination generates the dense carriers around the surface region. These carriers have the ability to cause the instantaneous surface potential modulation across the $\mathrm{GaSb} / \mathrm{GaAs}$ interface, which drives the coherent LO phonon even in the optically covered GaAs buffer layer.

\section{SUMMARY}

We have investigated the $\mathrm{THz}$ wave from the $\mathrm{GaSb} / \mathrm{GaAs}$ epitaxial structure. The Fourier power spectrum of the $\mathrm{THz}$ wave exhibits both the coherent $\mathrm{GaAs}$ and $\mathrm{GaSb}$ LO phonons. Note that the GaAs layer is optically masked by the top GaSb layer. The responsible factor for the generation of the coherent GaAs LO phonons has been attributed to the phenomenon that the instantaneous surface potential modulation originating from the impulsive carrier excitation by the pump pulse reaches the GaAs buffer layer, which triggers the coherent GaAs LO phonons.

\section{ACKNOWLEDGEMENT}

The present work was supported by Grant-in-Aid for Young Scientists B (No. 22760010) from the Japan Society for the Promotion of Science.

\section{REFERENCES}

1. For a review, P. H. Boliver, "Coherent terahertz spectroscopy" in Semiconductor Quantum Optoelectronics edited by A. Miller, M. Ebrahimzadeh, and D. M Finlayson, London: Institute of Physics, 1999, pp.151-192. References of THz spectroscopy are therein.

2. For a review, Terahertz Optoelectronics edited by K. Sakai, Berlin: Springer, 2005. References of THz spectroscopy are therein.

3. T. Dekorsy, H. Auer, C. Waschke, H. J. Bakker, H. G. Rokos, H. Kurtz, V. Wagner, and P. Grosse, Phys. Rev. Lett. 74, 738-741 (1995).

4. M. Tani, R. Fukasawa, H. Abe, S. Matsuura, K. Sakai, and S. Nakashima, J. Appl. Phys. 83, 24732477 (1998).

5. P. Gu, M. Tani, K. Sakai, and T. -R. Yang, Appl. Phys. Lett. 77, 1798-1800 (2000).

6. K. Mizoguchi, T. Furuichi, O. Kojima, M. Nakayama, S. Saito, A. Syouji, and K. Sakai, Appl. Phys. Lett. 87, 093102 1-3 (2005).

7. H. Takeuchi, J. Yanagisawa, S. Tsuruta, H. Yamada, M. Hata, and M. Nakayama, Jpn. J. Appl. Phys. 49, 082001 1-5 (2010).

8. H. H. Wieder, Surf. Sci. 132, 390-405 (1983).

9. T. Dekorsy, G. C. Cho, and H. Kurtz, "Coherent Phonons in Condensed Media" in Light Scattering in Solids VIII edited by M. Cardona and G. Güntherodt, Berlin: Springer, 2000, Chapter 4.

10. D. E Aspnes and A. A. Studna, Phys. Rev. B 27, 985-1009 (1983).

11. M. Nakayama, K. Kubota, T. Kanata, H. Kato, S. Chika, and N. Sano, J. Appl. Phys. 58, 4342-4345 (1985). 